\title{
Towards the Batch Production of 5 nm Focal Spot Size Zone Plates and Beyond
}

\author{
$\underline{\text { Nicolaie Moldovan }}^{1 *}$, Jonathan M. Logan ${ }^{1}$, and Ralu Divan ${ }^{2}$,
}

1. Alcorix Co., Plainfield, IL,

2. Argonne National Laboratory, Center for Nanoscale Materials, Argonne, IL, USA

* Corresponding author, moldovan@alcorix.com

Increasing the focusing capability of hard X-ray zone plates would enable many exciting discoveries in diverse scientific and technological fields, including drug design, nanotechnology, battery membranes, and supercapacitors. However, hard X-ray microscopy and tomography with Fresnel zone plates (FZPs) are limited in resolution to $\sim 25 \mathrm{~nm}$. High energy X-rays offer increased penetration depth and allow excitation in K-absorption edges of medium-heavy elements and in L and M absorption edges of heavy elements for chemical analysis. Currently, synchrotron radiation facilities are pursuing upgrades to increase the coherence and brilliance of their beam line sources, to create conditions for sub- $20 \mathrm{~nm}$ focusing. These conditions put a tremendous demand on fabrication of adequate FZPs, requiring Fresnel zones of few nm width and few $\mu \mathrm{m}$ height, with aspect ratios of 100:1 to 1000:1. For such aspect ratios, traditional top-down fabrication processes such as lithography and etching or electroforming are far beyond their limits. Bottom-up techniques such as sequential sputtering [1] or sequential atomic layer deposition onto wires [2] or into capillary lumens [3], followed by slicing have been proposed but of limited utility due to zone distortions or slicing difficulties. Here, we demonstrate a fabrication method capable of batch producing hard X-ray zone plates to form hundreds of device chips out of a single $\mathrm{Si}$ wafer. These zone plates show extraordinary potential to serve as hard X-ray zone plates for the focusing of X-rays to $5 \mathrm{~nm}$ and beyond.

While fabrication of a functional FZP would require 2-weeks-long depositions of hundreds of layers respecting the Fresnel zone width law, here we limit our experiment to 50 layers varying from $25 \mathrm{~nm}$ to 5 $\mathrm{nm}$ to prove the feasibility of controlled deposition and integration of the sequential ALD process with the subsequent batch processing. The first step is to fabricate cylindrical Si pillars on the wafer using the Bosch deep reactive-ion etching process (Fig. $1 \mathrm{a}, \mathrm{b}, \mathrm{c}$ ). The Bosch process results in precursor cylinders, each with a sidewall roughness of several hundred $\mathrm{nm}$, which must be smoothened before they can be used as templates for the growth of diffractive optics. Next, we coat the Si wafer with $350 \mathrm{~nm}$ coating of phosphorous-silicon glass (PSG) and perform a rapid thermal annealing process at $1150^{\circ} \mathrm{C}$ causing reflow of the PSG and achieving a sidewall roughness of $0.61 \mathrm{~nm}$ rms (Fig. $1 \mathrm{~d}$ ). Diffractive X-ray optics can be formed on the Si pillars through sequential ALD of two or more materials with contrasting optical properties, such as $\mathrm{Al}_{2} \mathrm{O}_{3}$ and $\mathrm{Ta}_{2} \mathrm{O}_{5}$. As a demonstration of this capability, we deposit a nanolaminate film of $\mathrm{Al}_{2} \mathrm{O}_{3}$ and $\mathrm{Ta}_{2} \mathrm{O}_{5}$ on precursor pillars (Fig. 2 b) using a MINI Chamber viscous flow hot wall ALD reactor (Vapor Pulse, LLC).

To serve as useful devices, the Si wafer with pillars containing the ALD nanolaminate films must be planarized, bonded to a carrier Si wafer, back side polished, and membranes have to be formed. For planarization, we deposit a $25 \mathrm{~nm}$ Pt ALD layer on the pillars to serve as a plating base and electroplate $\mathrm{Cu}$ with a thickness equal to the measured depth of the precursor structures, using a $\mathrm{CuSO}_{4} / \mathrm{H}_{2} \mathrm{SO}_{4}$ acid bath (Fig 2 a). The $\mathrm{Cu}$ material holds the Si pillars rigidly in place during polishing planarization. At the end, we attain $\sim 21 \mu \mathrm{m}$ thick Si membrane dies that are bonded to Si frames. Each die contains dozens of embedded nanolaminate devices. Figure $2 \mathrm{~b}$ shows a pillar after planarization and confirms that the 
nanolaminate film has not been deformed during planarization, showing the $5 \mathrm{~nm}$ layers intact. This demonstration illustrates that FZPs with remarkable parameters are possible by simply using the technique with longer ALD runs. Extensions of the technology for more-than-two ALD layers in the sequence may lead to approximations of graded index Fresnel kinoforms with close to $100 \%$ diffraction efficiency. Optimizing the design for maximum efficiency in higher order foci is also possible and would allow devices with sub-3-nm focusing or imaging resolution with possible refinements of the technique.

While the above prove the feasibility of the novel fabrication method using a surrogate nanolaminate, the functioning of the ultra-high aspect ratio FZPs is supported by the possibility to control the sidewall tilt to $0.1^{\mathrm{O}}$ on the precursor Si pillars, which will provide a required tilt condition for volume propagation effects. Also, given the limited extent of the zoned nanolaminate width, the tolerance on the central pillar diameter can be relaxed to about 3 finest zone widths (i.e. 10-30 nm) to still allow focusing with high efficiency. This explains how a resolution of $5 \mathrm{~nm}$ with multilayer FZPs made on wire was in fact possible. [4]

\section{References:}

[1] T. Koyama et.al., Rev Sci Instr. 83, (2012) p. 013705

[2] M. Meyer et.al., J. Synchrotron Radiation 20, (2013) p. 433

[3] J. H. Je, J. Jung, patent US 20130280421 A1, (2013)

[4] F. Döring et.al., Optics Express 21, 16, (2013) p. 19311

[5] This work was supported by the National Science Foundation under Grant No. 1648219. Any opinions, findings, and conclusions or recommendations expressed in this material are those of the authors and do not necessarily reflect the views of the National Science Foundation. Use of the Center for Nanoscale Materials, an Office of Science user facility, was supported by the U. S. Department of Energy, Office of Science, Office of Basic Energy Sciences, under Contract No. DE-AC02-06CH11357. Olga Makarova is thanked for contributions to the experimental work.
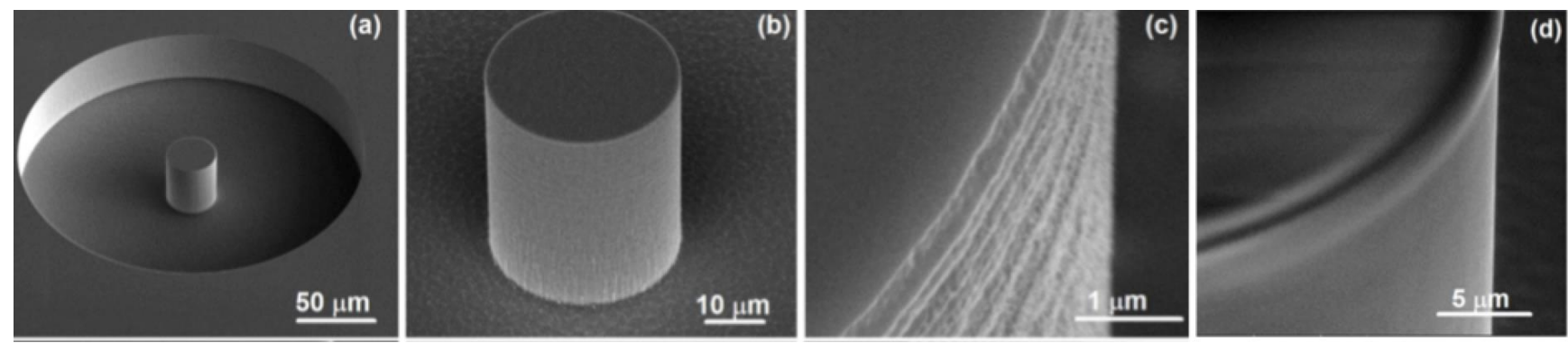

Figure 1. (a) SEM image of as-etched pillar; (b) detail of pillar; (c) side wall of pillar, showing as-etched roughness; (d) smoothing with $350 \mathrm{~nm}$ of PSG annealed at $1150^{\circ} \mathrm{C}$.

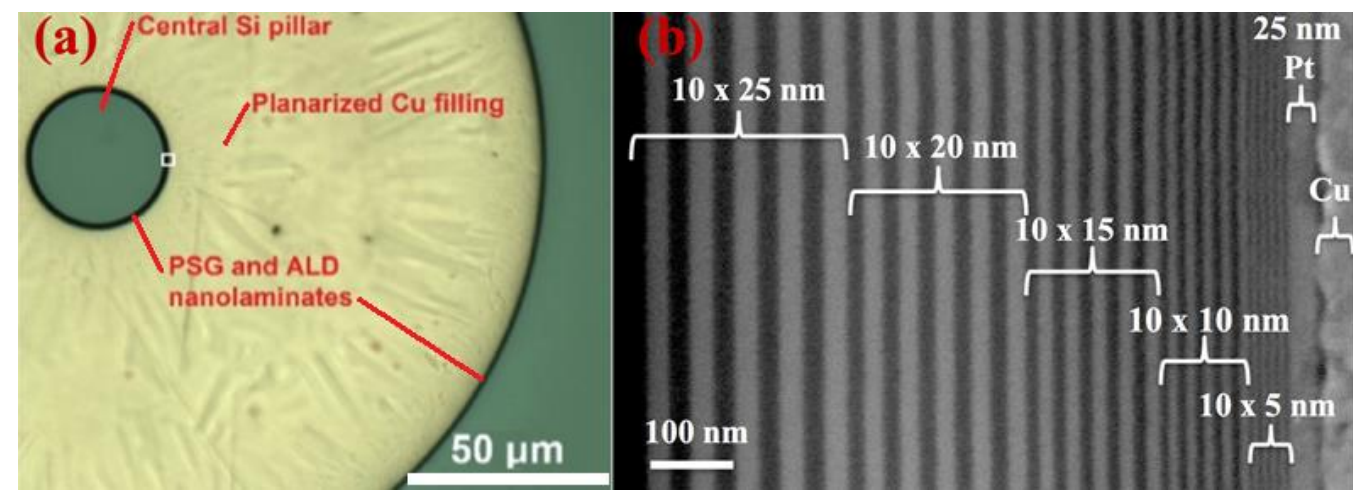

Figure 2. Si pillar with $\mathrm{Al}_{2} \mathrm{O}_{3} / \mathrm{Ta}_{2} \mathrm{O}_{5}$ nanolaminates after planarization; Optical microscopy view; (b) SEM image of nanolaminates on the edge of a Si pillar, with the 5-nm-thick layers visible. 